Special Issue of the 6th International Congress \& Exhibition (APMAS2016), Maslak, Istanbul, Turkey, June 1-3, 2016

\title{
A Study of the Dynamic Anterior Cervical Plate Implant by Computer Aided Virtual Engineering Method
}

\author{
Ö. KARAÇALI*
}

Istanbul University, Faculty of Engineering, Department of Mechanical Engineering, Avcılar, Istanbul, 34320 Turkey

\begin{abstract}
The convenience of dynamic anterior cervical PMMA biomaterial placement of spinal implants is considered by finite element modeling. The maximum fatigue behavior was also studied in surface coated Ti-5Al-2.55n spinal screw-rods. Anterior cervical biomaterial plates reduce the hazard for spinal cord injury and provide outstanding fixation for the anterior column, to stop the relocation and slackening of screws-rods by a cross-split screw crown that may be fastened into the biomaterial plate. This article reports about the hollow Ti-5Al-2.55n screw and cervical biomaterial PMMA plate system. The flexion movement of the spine implant was modeled to control the stresses and strains of the bone and screw interfaces under the external forces, as well as motion of the vertebrae by finite element modeling. This computational engineering analysis is aimed to support patients suffering anterior cervical arthrodesis after degenerative disease or trauma. The data from this research may provide an essential base to estimate the stabilization quality. A model for the biomaterial between C4 and C6 segments of vertebrae of cervical spine was obtained. Study of the cervical biomaterial implant provides instantaneous secure fixation virtual experiment with minimal complications, before the real implant surgery, using computer aided virtual engineering.
\end{abstract}

DOI: 10.12693/APhysPolA.131.584

PACS/topics: 87.10.Kn, 81.70.Bt, 87.15.La

\section{Introduction}

Anterior cervical plate is a mechanical device of spinal fixation to decrease the risk for spinal-neck-cord injury and provide outstanding fixation for the anterior column, to stop the relocation. It is found that the slackening of rod-screws occurs through a cross-split screw head that can be fastened into the biomaterial plate $[1,2]$. Vertebral compression in the back of the body causes 1.4 million fractures in the musculoskeletal system worldwide [3]. In this study, a most negative scenario of vertebrectomy situation was applied, where the implant inserts are embedded in vertebral body-like pieces and need to bear presso-flexion. The bone quality of spine determines the characteristics of the fixation screws and stabilizer plate implants [4]. Spinal implant systems for posterior fixation must be made of a good biologically responsive biomaterial and have biomechanical stability [5]. Biomechanical computational models of the spine implants had not included the developed nonlinear stresses and viscoelastic material properties [5-7]. Therefore, current simulation research of dynamic anterior cervical biomaterial plate (DACBP) placement was performed for computation of strain and stresses developed within the cervical spine area during several back motions of natural spine, by structural analysis method. The methods used for testing spinal instrumentation require a relatively long time for valid results of the new or improved implants [8]. Hence, the aim of the study is to determine the fatigue life of available commercial devices. The simulation of cyclic fatigue test method reduces the time

\footnotetext{
*e-mail: ozdogank@istanbul.edu.tr
}

used for testing the biomechanical properties of the spinal rods [9]. A virtual implant was developed to test the design of spinal implant. The implant under research was made from poly (methyl-methacrylate) (PMMA) biomaterial. The disc is of symmetrical design with screwable attachments on its top and bottom surfaces. The disc is thus sandwiched between rigid plates. The goal of the analysis was to accurately simulate flexion, extension, torsion and bending deformation modes. Heightening of anterior cervical plates, containing units that turn under compression, was modeled as in Fig. 1.

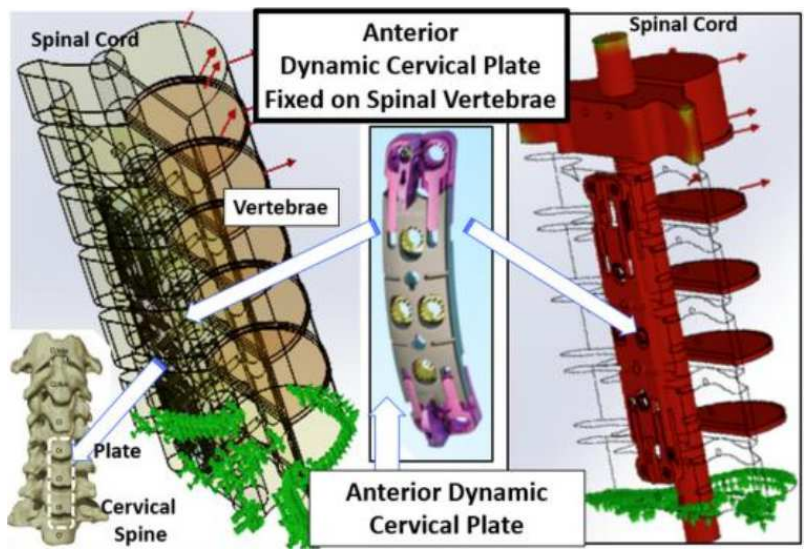

Fig. 1. Cervical plate and spinal vertebrae.

\section{Analysis of dynamic anterior cervical biomaterial plate}

Multi-step analysis was carried out by first simulating the pre-compression and then, by simulating the extension and flexion in the second step. This study takes into account the non-linear biomaterial properties of the 
PMMA biomaterial and large expansion deformations. The parametric constrained part model of DACBP-back spinal fixation insert was designed according to ASTM F1717 standards. The experimental static test was employed for spinal rod-screw to validate strains occurred. The simulation of spinal implants was performed considering mechanical properties of biomaterial, to assess functioning of implant under specific conditions. Also, dynamic stabilization and movement maintenance are considered and finite-element models of the spine are validated. The spine implant was examined for failure mechanisms and distortions. The finite element model (FEM) has included representative topology with cervical spine features, that consists of important motion and stiffness models with biomaterial non-linearity. The validity of the model was tested by increasing the number of cycles to break the $3.0 \mathrm{~mm}$ cross section diameter threads.

\section{Results}

Load forces ranging from $25 \mathrm{~N}$ to $400 \mathrm{~N}$ were applied during simulation according to the (ISO) 18192 standard and ASTM. Test simulations have shown that these unidirectional plates needed less movement and clinched alongside flexion-extension, shown in Fig. 2. It was also observed that an additional reliable graft load was seen when contrasted with bidirectional configuration.

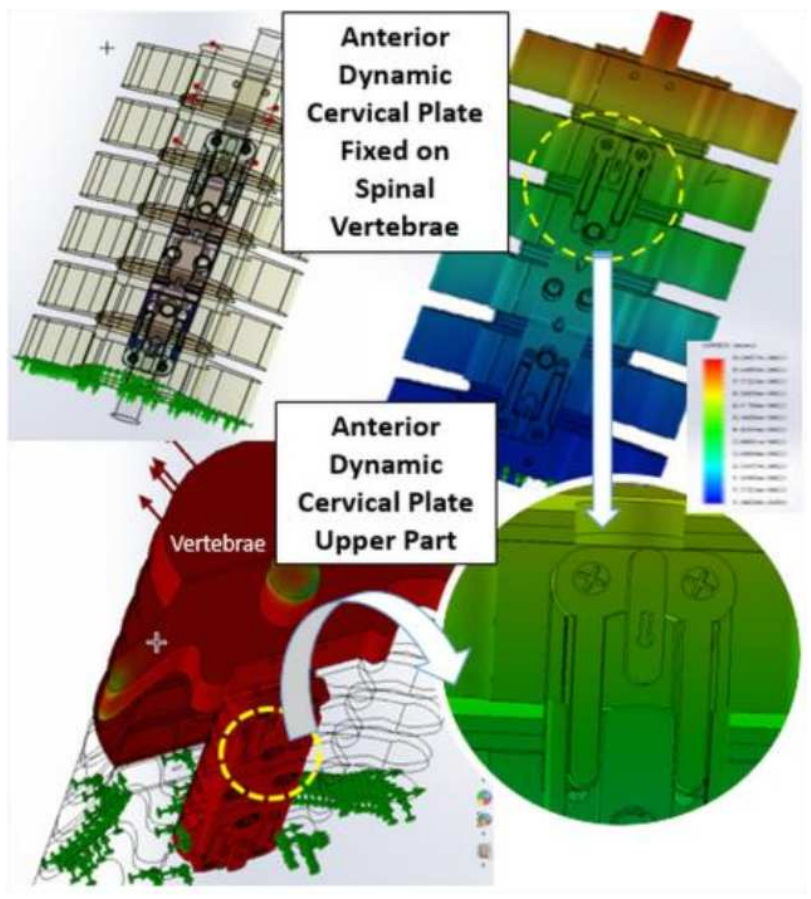

Fig. 2. DACBP system assembly.

Unidirectional movement of the DACBP was permitted up to $2 \mathrm{~mm}$ of unidirectional translation for every level to $1 \mathrm{~mm}$ additions. Unidirectional development results in a constant compressive load between the end plate and the bone implant, in light of nonclinical testing. $4500 \mathrm{~N}$ mm flexion moment, $350 \mathrm{~N}$ compressive load and $350 \mathrm{~N}$ tension load were applied to predict the behavior of the model. The stresses under given loads are shown in Fig. 3. The moment and forces were applied to vertebra surfaces of C4 and C6. The loading of the segments was accomplished by ANSYS program. ANSYS version 17.2 is used in the analysis. Then, a precise parametric insert analysis of screw head and rod was realized by Von Mises stress expansion. Maximal stress level was over $24 \mathrm{MPa}$ in flexion situation. The flexion accompanied by stretching are seen as the cause of change in DACBP behavior for the period of simulated performance of spine. During simulation, maximum stress level occurred in the implant rod-screw zone as shown in Fig. 3.

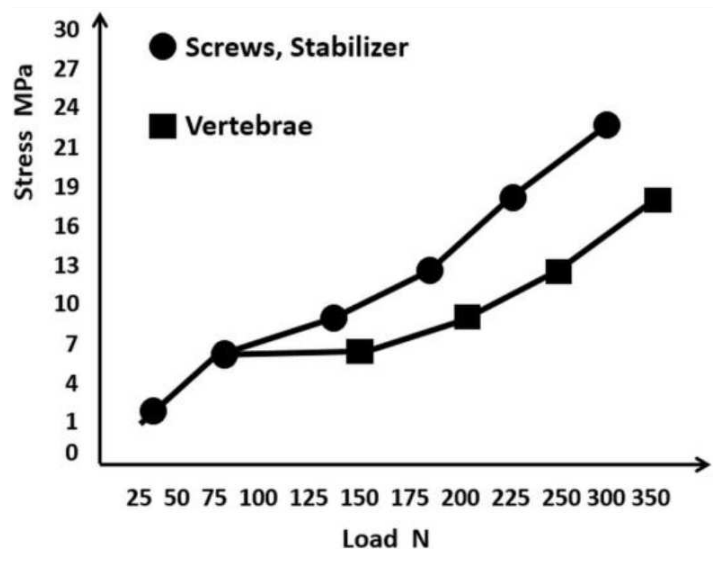

Fig. 3. Stresses during loading of DACBP.

In this research, equivalent Von Mises yield criterion [10] for elastic stress Eq. (1) and strain Eq. (2) were computed from the integrated screw teeth and bone area. Computational simulation software has employed the $v^{\prime}$, the effective Poisson's ratio. The bone and thread strain were checked to see if the bone was strong enough. The equivalent stresses occurred in the $\mathrm{C} 4$ and $\mathrm{C} 6$ vertebrae are shown in Fig. 3.

$$
\begin{aligned}
& \sigma_{\text {Von Mises }}=\left(\frac { 1 } { 2 } \left[\left(\sigma_{1}-\sigma_{2}\right)^{2}+\left(\sigma_{2}-\sigma_{3}\right)^{2}\right.\right. \\
& \left.\left.+\left(\sigma_{3}-\sigma_{1}\right)^{2}\right]\right)^{1 / 2} \\
& \sigma_{\text {Von Mises }}=\frac{1}{1+v^{\prime}}\left(\frac { 1 } { 2 } \left[\left(\varepsilon_{1}-\varepsilon_{2}\right)^{2}+\left(\varepsilon_{2}-\varepsilon_{3}\right)^{2}\right.\right. \\
& \left.\left.\quad+\left(\varepsilon_{3}-\varepsilon_{1}\right)^{2}\right]\right)^{1 / 2} .
\end{aligned}
$$

The parametric analysis of screw head shows that the unsupported screw length and the cantilever arm parameters are the most stressed regions. Cyclic fatigue life testing simulation was applied at 290 and $440 \mathrm{~N}$ force levels. During experiment the displacements of biomaterial stabilizer and metallic parts of Ti-5Al-2.55n were $0.009 \mathrm{~mm}$. Displacements for the biomaterials did not exceed the value of $0.12 \mathrm{~mm}$. This analysis shows that the 
stiffness and stability of DACBP system within the loading limits is acceptable. The stabilizer and screws maximum equivalent stress levels were evaluated as $24 \mathrm{MPa}$. The C4-C6 segments were subjected to $2000 \mathrm{~N}$ load by stabilizer. On the other hand the applied stress induced in the vertebral parts has exceeded $180 \mathrm{MPa}$.

The computational analyses were carried out using the up-to-date modeling ANSYS software. The compressive testing, static tensile and cyclic fatigue testing were accomplished in the experimental work. ASTM F451-99a standards were used for static tensile and compressive testing, while ASTM standard F1717 was used for cyclic fatigue testing in 13-25 MPa stress ranges. As a finding of this research, the biomaterial strength of the DACBP was assessed by investigation of static mechanical properties and fatigue life.

The static test results were obtained in this study. The DACBP cyclic fatigue test simulation was conducted with loading up to $1 \mathrm{MHz}$ to construct the Wöhler diagram. There is a good agreement between the standard and these findings (Fig. 4).

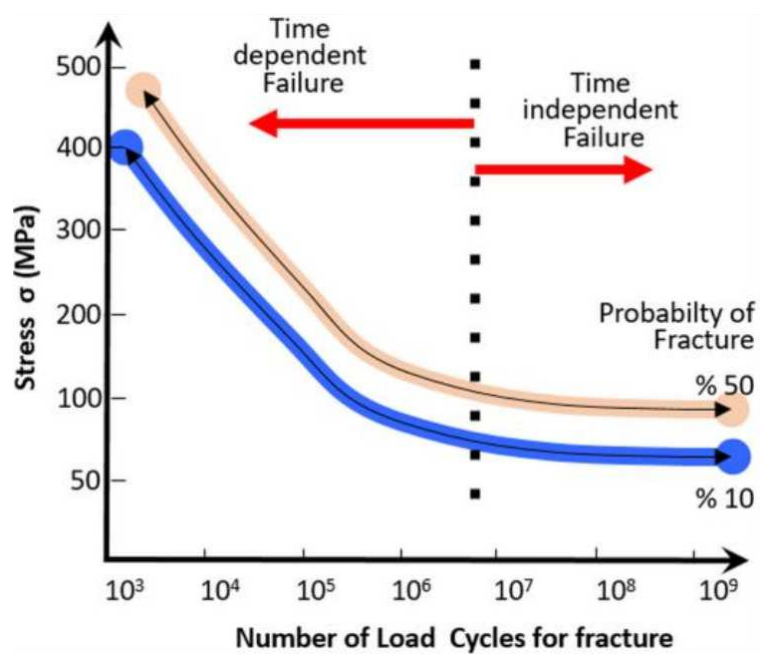

Fig. 4. The DACBP S-N cyclic fatigue test.

\section{Discussion}

The main outcome of this research was the excessive motion at the lower-end vertebra-screw junction in the DACBP model, caused by stresses, strain and fatigue. The developed FEM is suitable for analysis of dynamic cervical plate under rapid flexural motions. Morcher has initially stated the utilization of the securing plate in the cervical spine in 1986 [11]. After that, problems of plating, particularly releasing of the screws and the plates have diminished extraordinarily [12].

In the study of Lazennec et al. [13], the fusion rate was higher with dynamic plates. To be able to compare the results, the presented system has used a similar fusion rate for extendable wings. Previous studies of spinal implants did not consider the extendable parts that influence the results of the forces, according to their sites, number and shapes [14].
The link between spinal DACBP implant and the rod was the only link between extensions as mechanical joint. The simulations were more precise and reproducible than those in cadaver models [15]. Nevertheless, the experiment outcomes cannot predict the actual situation of in vivo application of the modified DACBP system. In the literature, the complications concerning the front cervical plate are restricted to releasing or breaking of the equipment and tracheoesophageal or neurovascular auxiliary wounds [16]. Different sorts of complications that may prompt to clinical indications are overlooked, and the emphasis is fundamentally made on unconstrained plates [17]. Furthermore, there are few reports concerning an extensive example estimate $[5,12,13]$. A great amount of difficulties experienced with cervical bolting plates are considered whereas only flexural and tensile mechanical phenomena are studied in these investigations. The DACBP system was implemented according to the ASTM F1717 testing standard to imitate standardized dynamic anterior cervical plate to analyze its biomechanical properties [17].

The DACBP system has provided positive results and it is seen that using dynamic plates is so advantageous, that it can be later used in the clinical settings. One significant limitation of this study is that the calculation of applied moments is prone to error, given the large loads and small moments imposed in these virtual experiments. In summary, the outcomes of this research show promising results for the application of expandable biomaterial parts of the cervical spine. Additional studies are necessary to examine the contradictory data concerning biomechanical efficiency of the expandable parts of the DACBP.

\section{Conclusions}

Analysis of the cervical biomaterial implant provided instantaneous secure fixation virtual experiment with minimal complications, before a real implant surgery, using computer aided virtual engineering. In this research, mathematical modeling of biomechanical anterior cervical plate was applied to determine the stresses, strains and displacements during applied loading by FEM. The C4-C6 vertebrae units were simulated using real load conditions.

The ASTM F1717 standards were used for DACBP experimental system to realize the biomaterial and procedures of static and cyclic fatigue testing in the virtual environment. A new model was developed by design variables and fatigue behavior analysis. The cyclic fatigue life of DACBP system was computed by S-N fatigue curve after FEM analysis. The results have revealed that as the load applied to the system is increased, the fatigue life decreases. The outcomes of the research for spine implant between $\mathrm{C} 4-\mathrm{C} 6$ vertebral parts were very valuable in deciding the characteristics of the DACBP stabilizer and cervical plate-PMMA in case of choosing the metallic biomaterial. 


\section{Acknowledgments}

This research is funded by Istanbul University, Turkey, projects No: 23472 and 24431 (The Scientific Research Foundation). Such support is greatly acknowledged.

\section{References}

[1] T.L. Johnston, E.E.K. Karaikovic, E.P. Lautenschlager, D. Marcu, Spine J. 6, 667 (2006).

[2] M.A. Davies, S.C. Bryant, S.P. Larsen, D.B. Murrey, D.S. Nussman, E.B. Laxer, B.V. Darden, J. Biomech. Eng. 128, 481 (2006).

[3] L.L. Barbera, F. Galbusera, T. Villa, F. Costa, H.-J. Wilke, Proc. IMech E. Part H: J. Eng. Med. 228, 1014 (2014).

[4] S. Kurtz, M. Villarraga, K. Zhao, A. Edidin, Biomaterials 26, 3699 (2005).

[5] P. Schleicher, R. Gerlach, B. Schar, C.M.J. Cain, W. Achatz, R. Pflugmacher, N.P. Haas, F. Kandziora, Eur. Spine J. 17, 1757 (2008).

[6] J.A. Beltran-Fernández, L.H. Hernández-Gómez, G. Urriolagoitia-Calderón, A. González-Rebatú, G. Urriolagoitia-Sosa, Appl. Mech. Mater. 24-25, 287 (2010).

[7] C.M. DuBois, P.M. Bolt, A.G. Todd, P. Gupta, F.T. Wetzel, F.M. Phillips, Spine J. 7, 188 (2007).
[8] D.S. Korres, I.S. Benetos, D.S. Evangelopoulos, M. Athanasssacopoulos, P. Gratsias, O. Papamichos, G.C. Babis, Eur. J. Orthop. Surg. Traumatol. 17, 521 (2007).

[9] R.R. Campos, R.V. Botelho, Eur. Spine J. 23, 298 (2014).

[10] T. Brandt, M. Morcher, I. Hausser, Cerebral Artery Dissection 20, 16 (2005).

[11] D. Korres, V.S. Nikolaou, M. Kaseta, D. Evangelopoulos, K. Markatos, J. Lazarettos, N. Efstathopoulos Eur. J. Orthop. Surg. Traumatol. 24, 125 (2014).

[12] J.Y. Lazennec, A. Aaron, O. Ricart, J.P. Rakover, Eur. J. Orthop. Surg. Traumatol. 26, 9 (2016).

[13] M.J. Fagan, S. Julian, A.M. Mohsen, Proc. Instn. Mech. Engrs. 216, 291 (2002).

[14] C.P. Yen, T.Y. Hwang, C.J. Wang, S.L. Howng, Acta Neurochir. 147, 665 (2005).

[15] M. Driscoll, J.-M.M. Thiong, H. Labelle, S. Parent, Bio. Med. Res. Int. 2013, 931741 (2013).

[16] L.L. Barbera, F. Galbusera, T. Villa, F. Costa, H.-J. Wilke, Proc IMechE Part H: J. Eng. Med. 228, 1014 (2014).

[17] J.-B. Park, Y.-S. Cho, K.D. Riew, J. Bone Joint Surg. Am. 87, 558 (2005). 